\title{
Non-Traumatic First Rib Fractures Secondary to Opposing Muscle Contractions: A Case Series
}

\author{
Jamie L Weis* \\ Department of Orthopaedic Surgery, St. Luke's University Health Network, USA
}

*Corresponding author: Jamie L. Weis, PA-C, DHSC, Department of Orthopaedic Surgery, St. Luke's University Health Network, 801 Ostrum St, Bethlehem, PA 18015, USA, Tel: 484-239-4274, Fax: 484-526-2541

\begin{abstract}
While first rib fractures typically occur from direct, traumatic injuries and have high rates of serious complication, less commonly, non-traumatic first rib fractures are encountered in young athletes. This paper presents the cases of a teenage basketball player with a first rib fracture after stretching, and that of a teenage cheerleader with a first rib fracture from straining to lift another cheerleader. Both patients became asymptomatic following conservative treatment and returned to sports. These cases were then evaluated next to the existing literature. The mechanism for these non-traumatic fractures is thought to be due to sudden contractions of opposing muscle forces on developing bone. Because of the biomechanics of the first rib's opposing muscle attachments, first rib fractures should be considered in patients who report pain deep to the medial clavicle, even without a history of direct, severe trauma.
\end{abstract}

\section{Keywords}

Rib fracture, Stress fracture, First rib

\section{Abbreviations}

CT: Computed Tomography; MRI: Magnetic Resonance Imaging; NSAIDs: Non-steroidal Anti-Inflammatories

\section{Introduction}

The first rib, a well-protected bone fully surrounded by other bony and muscular structures is not commonly fractured except in cases of direct, severe trauma, which carries a risk of serious visceral, vascular, or neurologic associated injuries. Even less common are stress fractures of the first rib from chronic opposing forces or non-traumatic fractures from a sudden contraction of opposing musculature. The cases presented below are those of a teenage female basketball player with a first rib fracture after stretching, and that of a teenage female cheerleader with a non-traumatic first rib fracture after straining to lift another cheerleader.

\section{Case Presentations}

\section{Case Report 1}

A 16-year-old female basketball player presented to the emergency department with acute upper trapezius pain. She had been having some soreness in the area for approximately two weeks. This was very mild, and she was able to ignore it easily even while playing basketball. Then after a game, she was stretching her arm downward with her cervical spine flexing posteriorly and to the contralateral side to try to crack her neck, when she felt a pop and sudden, sharp 10/10 pain in the triangle between the scapula, clavicle, and cervical spine. Her acute-on-chronic pain did not radiate, was not associated with numbness, and was not associated with any pulmonary symptoms. She was taken to the emergency department immediately where a shoulder $x$-ray revealed an acute, non-displaced fracture of the posterolateral aspect of the first rib (Figure 1).

She presented to orthopedics four days later in a sling. Her pain had improved to $0 / 10$ at rest and $4 / 10$ with active full shoulder flexion or full abduction. She had a full active range of motion of the cervical spine and shoulder. Past medical, surgical, and social history were unremarkable, and her vital signs were all within the normal range.

She had moderate tenderness to palpation in the upper trapezius area just deep to the medial aspect of the right clavicle and mild tenderness along the medial scapular border. Cardiopulmonary and neurological

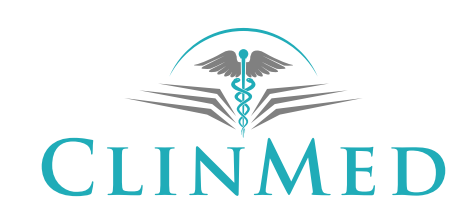

INTERNATIONAL LIBRARY

Citation: Weis JL (2019) Non-Traumatic First Rib Fractures Secondary to Opposing Muscle Contractions: A Case Series. Int J Sports Exerc Med 5:113. doi.org/10.23937/2469-5718/1510113

Accepted: January 10, 2019; Published: January 12, 2019

Copyright: (C) 2019 Weis JL. This is an open-access article distributed under the terms of the Creative Commons Attribution License, which permits unrestricted use, distribution, and reproduction in any medium, provided the original author and source are credited. 


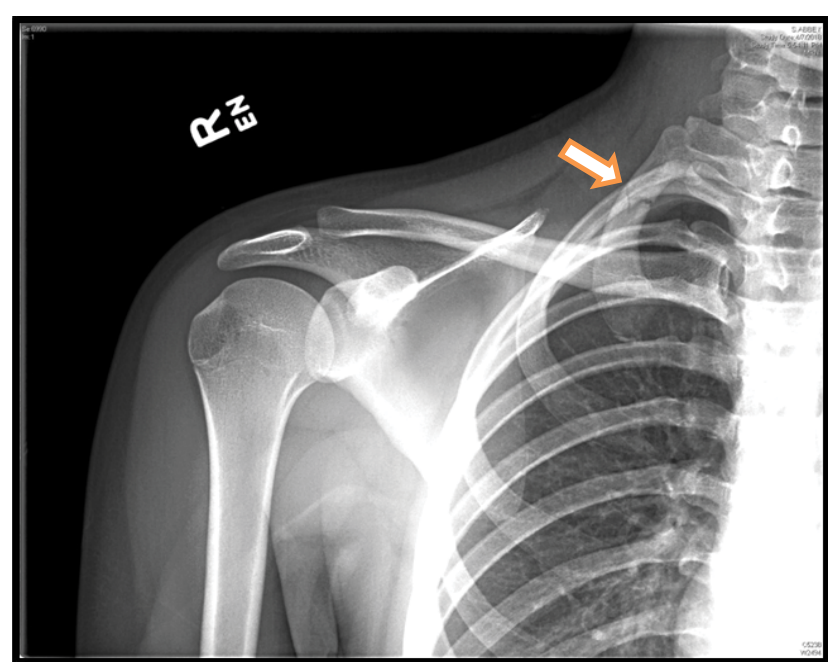

Figure 1: First rib fracture from Case 1.

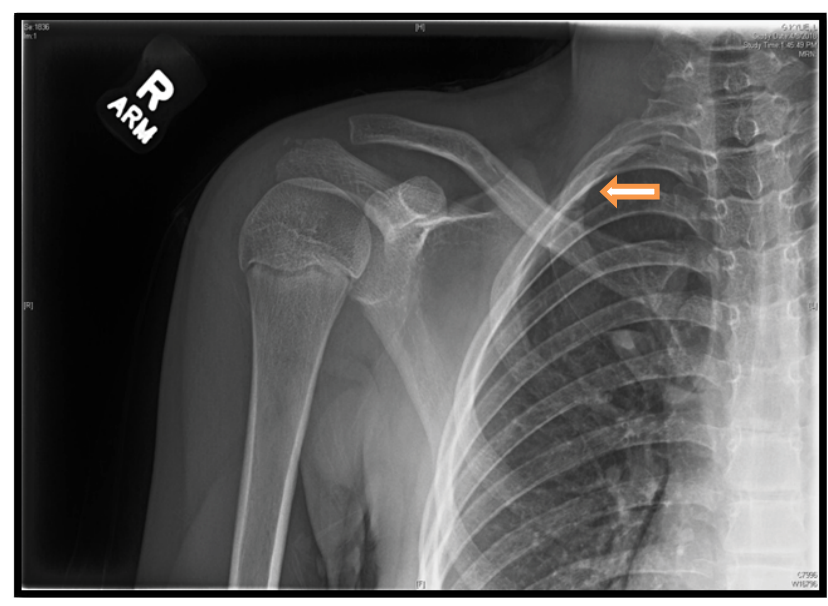

Figure 2: First rib fracture from Case 2.

exams were normal.A complete blood count, complete metabolic profile, alkaline phosphatase, vitamin $D$, and parathyroid hormone levels were all within the normal range.

\section{Case Report 2}

An 11-year-old female cheerleader presented to the orthopedic office with right shoulder pain. She had reached downward and grasped another cheerleader's leg to help lift her. During this maneuver, her arms had and inferior traction force applied and she strained her neck posteriorly and laterally. She felt a pop in her shoulder and had immediate pain. She had been completely asymptomatic before this moment. X-rays in the emergency department revealed an acute first rib fracture. Figure 2 She had normal vital signs and a normal shoulder exam with a full active range of motion and no laxity. She had some tenderness in the area of the superior, medial scapula.

Both patients were treated with a period of rest, activity modification including an overhead reaching restriction, and NSAIDs as needed. At the 12-week mark, the fracture line was still apparent on the basketball player's x-ray but by the 15 week mark, she was completely asymptomatic and returned to sports. The cheerleader in the second case had a complete resolution of symptoms by the two week mark. She started physical therapy for strengthening and returned to cheerleading.

\section{Discussion}

\section{Incidence}

Instances of non-traumatic first rib fractures have been reported in athletes participating in swimming $[1,2]$, baseball $[3,4]$, football $[5,6]$, soccer [3], tennis [6], powerlifting [7], rowing [8], lacrosse [9], martial arts [10,11], basketball [10], and surfing [12]. Many of these athletes participated in weight training for their respective sport $[1,2,7]$. Athletes with non-traumatic first rib fractures frequently participate in sports with repetitive overhead arm movements or heading (soccer) movements $[1,2,4,7,9,11,13]$.

While most of the articles in the literature focusing on non-traumatic first rib fractures are case studies, one older, retrospective study evaluated 1600 chest $\mathrm{x}$-rays originally done as tuberculosis screens and found eight $(0.005 \%)$ first rib fractures [14]. Another study retroactively reviewed 77,607 chest $x$-rays of U.S. Navy personnel and found $73(0.001 \%)$ first rib fractures [15]. One study on elite-level rowers found that rowers who had non-traumatic first rib fractures were more likely than their elite-level peers to have low bone mineral density, although their bone mineral density averages were equal to that of the general population [8]. Nontraumatic first rib fractures, resulting from either chronic stress over time $[3,5,6,11]$ or from a sudden, forceful contraction of opposing muscles $[1,4,6,9,11]$ are exceedingly rare. They tend to occur in adolescent or teenage athletes, perhaps due to bony maturation lagging behind muscle development [16].

\section{Anatomy and Physiology}

The first rib is a small, flat rib with a $180^{\circ}$ arc from the T1 vertebra to the manubrium. On the flat, superior surface, are two shallow grooves, one for the subclavian artery and one for the subclavian vein, between which is the scalene tubercle for the scalene muscle attachment. On the inferior surface the serratus anterior and intercostal muscles attach. The scalene muscles pull superiorly and the serratus anterior and intercostals pull inferiorly on the first rib at these subclavian grooves $[1,4,9,10,11]$. Figure 3 Originally labeled Surfer's Rib in 1985 , in the absence of direct sudden trauma, the first rib tends to fracture at the subclavian grooves, the weakest point, when these opposing muscles are both suddenly and forcefully engaged [12].

\section{Literature and evaluation}

While this can be a repetitive stress injury with insidious onset, it can also present as sudden, acuteon-chronic symptoms as the stress fracture becomes 


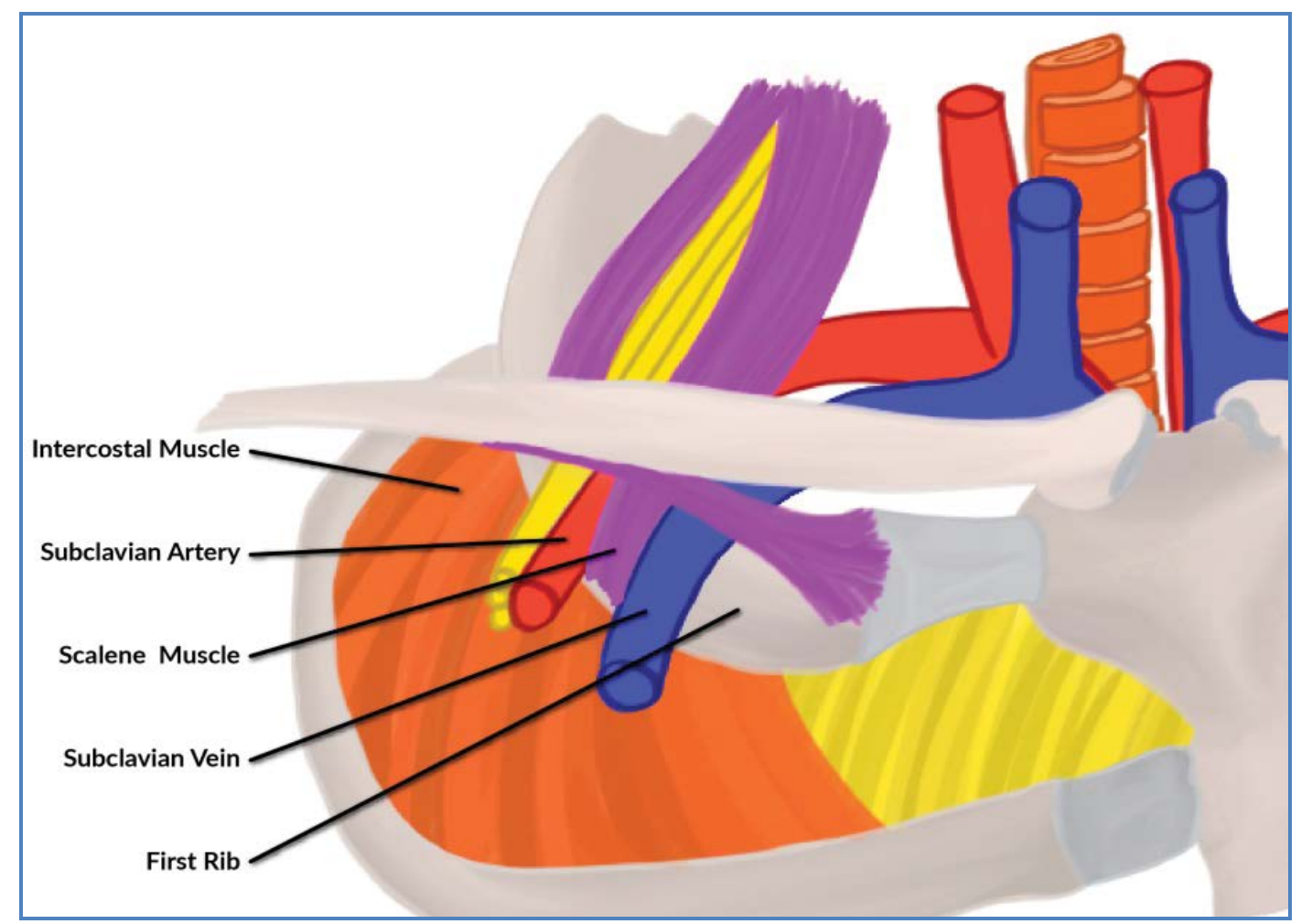

Figure 3: First rib anatomy.

a complete fracture as demonstrated by the basketball player in case 1 . These fractures can also present as cases of sudden, acute pain with no prior symptoms as seen in case 2 . This sudden muscle contraction mechanism involves pulling from a posterolaterally extended neck with traction on the contralateral arm [1] as described in the original surfer's layback position case [12], and in the two current cases of the basketball player stretching to crack her neck and the cheerleader lifting another cheerleader.

Similar to the current cases, patients generally present with a full range of motion of the neck and shoulder $[1,3,4]$, mild shoulder pain with flexion or abduction $[3,4,9,11]$, and nearly all had point tenderness in the upper shoulder deep to the medial clavicle and the scapula $[3,4,6,9,10,11]$. Some presented with transient pleuritic chest pain [1,11], and one case describes scapular winging [1]. Neither of the two athletes above demonstrated chest pain, pulmonary symptoms, or scapular dysfunction.

Generally, plain x-rays of either the chest or shoulder will show the first rib fracture. However, radiographs can miss this fracture, so if there is clinical suspicion, CT scan, MRI, and triple phase bone scan are all more sensitive $[1,6,7,15]$. Given the location of the fracture, a careful neurovascular exam is warranted.

Patients are typically treated with rest, activity modification to restrict overhead reaching, and NSAID analgesics. Physical therapy, as prescribed in case 1 , is commonly used for shoulder motion and strengthening $[1,6]$. One case reported using a bone stimulator [3].
Rarely, in prolonged, complicated cases, surgical first rib resection can be done for symptomatic relief $[5,6,11]$.

Without trauma, there is an extremely low incidence of associated neurological, vascular, or visceral complications. There was one report of thoracic outlet symptom secondary to excessive callus formation at the fracture site [5], one case of sternoclavicular subluxation [6], and one case of torticollis in a child [10]. Horner's syndrome as a consequence of first rib fracture has been described, but never in a non-traumatic fracture case [13]. The most common complication is pseudarthrosis or non-union, however these patients, like the athlete in case 1 , have generally been asymptomatic despite the pseudarthrosis and have returned to sport successfully $[1,4,6,9,11,17]$. This case series brings attention to an injury pattern in young athletes that though uncommon, is well-documented and could be overlooked on shoulder or chest $\mathrm{x}$-ray without deliberate consideration.

\section{Conclusion}

Fractures of the first rib are particularly concerning with regards to the potential for associated subclavian vessel, brachial plexus, or lung and pleural injury. While related complications have been reported in traumatic first rib fracture cases, acute injury to these structures has not been reported in non-traumatic first rib fractures. Due to the anatomy of the first rib with opposing serratus anterior, intercostal, and scalene muscle attachments bracketing the subclavian grooves, first rib fracture should be considered in patients, particularly teenage athletes, presenting with subclavicular pain, even in the absence of severe, direct trauma. 


\section{Acknowledgment}

The author would like to thank Karen Ruggles, MS, assistant professor of computer science, DeSales University, for the medical illustration.

\section{Funding Statement}

The author received no funding for this case study.

\section{Statement of Equal Contribution}

N/A.

\section{References}

1. ALDERSON BR (1947) Further observations on fracture of the first rib. Br J Radiol 20: 345-350.

2. Bailey $P$ (1985) Surfer's rib: isolated first rib fracture secondary to indirect trauma. Ann Emerg Med 14: 346-349.

3. Blichert-Toft M (1969) Fatigue fracture of the first rib. Acta Chir Scand 135: 675-678.

4. Eng J, Westcott J, Better N (2008) Stress fracture of the first rib in a weightlifter. Clin Nucl Med 33: 371-373.

5. Lee SJ, Yie K, Chon SB (2012) Juvenile first rib fracture caused by morning stretching. J Emerg Med 43: 119-121.

6. Lin YC, Chuang MT, Hsu CH, Tailor AR, Lee JS (2015) First Rib Fracture Resulting in Horner's Syndrome. J Emerg Med 49: 868-870.

7. Low S, Kern M, Atanda A (2016) First-rib stress fracture in two adolescent swimmers: a case report. J Sports Sci 34: 1266-1270.

8. Matsumoto T, Fujita K, Fujioka H, Tsunoda M, Yoshiya S, et al. (2003) Stress fracture of the first rib in a soccer player: a rare etiology of shoulder pain. J Shoulder Elbow Surg 12: 197-199.

9. Mirza AK, Duncan AA1 (2017) Thoracic outlet syndrome as a consequence of isolated atraumatic first rib fracture. $\mathrm{J}$ Surg Case Rep 2017: 100.

10. Nguyen HT, Carmichael JP, Bainbridge JS, Kozak C (2006) First rib fracture of unknown etiology: a case report. $J$ Manipulative Physiol Ther 29: 590-594.

11. O Neal M, Ganey T, A Ogden J (2009) First rib stress fracture and pseudarthrosis in the adolescent athlete: The role of costosternal anatomy. Clin J Sport Med 1: 65-67.

12. Papadimitriou NG, Christophoridis J, Papadimitriou A, Beslikas TA (2005) Acute torticollis after isolated stress fracture of the first rib in a child. A case report. J Bone Joint Surg Am 87: 2537-2540.

13. Sakellaridis T, Stamatelopoulos A, Andrianopoulos E, Kormas $\mathrm{P}(2004)$ Isolated first rib fracture in athletes. $\mathrm{Br} \mathrm{J}$ Sports Med 38: e5.

14. Taimela S, Kujala UM, Orava S (1995) Two consecutive rib stress fractures in a female competitive swimmer. Clin $\mathrm{J}$ Sport Med 5: 254-256.

15. Vikramaditya $P$ (2001) Two cases of isolated first rib fracture. Emerg Med J 18.

16. Vinther A, Inge-Lis K, Christiansen E, Alkjaer T, Larsson B, et al. (2005) Exercise-induced rib stress fractures: Influence of reduced bone mineral density. Scand J Med Sci Sports 15: 95-99.

17. Wild A, Begly JP, Garzon-Muvdi J, Desai P, McFarland EG (2011) First-rib stress fracture in a high-school lacrosse player: A case report. Sports Health 3: 547-549. 